\title{
Study of Personalized Trust Model in Enterprise Computing Environment
}

\author{
Liu Lu and Zhu Yanchun \\ School of Economics and Management, BeiHang University, 100083 \\ Beijing, China, \\ liulu@buaa.edu.cn, waterflour@163.com
}

\begin{abstract}
The increasing popularity of resource exchange through peer-topeer networks has encouraged the development of ways to support distributed and heterogeneous enterprise computing. Unfortunately, the prospect of distrust attracts agents seeking to weaken the network by propagating fraud and bad services, that is, establish the trust mechanism of the P2P network. Based on recommendation, a number of computational trust and reputation models were proposed for resource-sharing network based on agents. This paper presents a recommendation-based trust model, which utilizes theory of collaborative filtering and social network. Compared with existing trust model, this model has two main features. First, it eliminates malicious recommendation by evaluating credibility of recommender. Second, it adjusts calculating of path weight, while combining trust path, which addresses the problem of subjectivity in path weight initialization. Finally, comparing with Beth model based on eBay's data, the result shows the feasibility and effectiveness of this approach.
\end{abstract}

\section{Introduction}

The rapid development $o$ of internet and enterprise computing has stimulated the use of resource sharing and exchange using peer-to-peer (P2P) networks in distributed and heterogeneous enterprise computing. However, the accessibility of these systems makes them vulnerable to malicious users wishing to poison the system with corrupted data or harmful services for personal. The lack of trust mechanism has resulted in large numbers of frauds and bad services [1].

Many research groups and organizations have proposed trust models based on recommendation, applied in resource sharing service of the $\mathrm{P} 2 \mathrm{P}$ pattern successfully. In recommendation-based trust model, recommending trust coalition of trust path is the key best of all. Analyzing existing recommend-based trust model, there have

Please use the following format when citing this chapter:

Lu, L., Yanchun, Z., 2006, in International Federation for Information

Processing, Volume 205, Research and Practical Issucs of Enterprise Information Systems, eds.

Tjoa, A.M., Xu, L., Chaudhry, S., (Boston:Springer), pp.443-448. 
been a great deal of efforts in trust quantification, but still not enough on trust transmission.

The Beth [2] model utilizes probability to model subject trust in brief and synthesize recommendation-based trust by getting mean, which can't reflect truth of the trust relationship. The model by Rahaman [3] just figures out the transmission rule and calculating formula of trust degree. So it can't deduce recommendationbased trust. There are also many other scholars, who adopt variant means to measure and deduce the trust relationship, for example the Bayes network [4], rough set theory [5], evidence theory [6,7], social network analysis [8], probability [2], etc. However, all these models have some problems in the aspect of trust transmission. There exist many limitations in trust coalition, which mostly include the high subjectivity in setting weight, difficult to reflect the trust reality and unable to eliminate negative impacts of malicious recommending.

The objective of this study is to apply recommendation theory to trust calculating. In the social network, trust relationship is the key of users' human relations and the trust degree of two strangers is usually decided by recommendation of other users. This paper presents a recommendation-based trust model, which utilizes theory of collaborative filtering and social network.

\section{Recommendation-Based Personal Trust Model}

In our model, users will select those who have transaction history with unknown user and has close trust scenario and trust tendency as the recommender of that unknown user. Then based on the recommendation of above recommender, users can build parallel trust network and incorporate trust paths, finally get the personal evaluation of unfamiliar user's trust.

Following is the sequence of our model: 1) indirect trust computing.2) trust path deduction. 3) trust path amalgamation. 4) trust computing of unfamiliar user.

\subsection{Indirect Trust Computing}

Aiming at the limitation of model proposed by Beth [2] and Yu [7], the paper sets the maximum length of a trust path as 6 according to Six Degrees of Separation suggested by social psychologist Stanley Milgram [5], which cuts down the time of traversing in trust paths, therefore, raises computing efficiency and strengthens explainability of trust model by using existing research of sociology theories.

Definition 1 The direct trust $\tau_{i}^{j}$, which user $i$ hold about user $j$ is given by

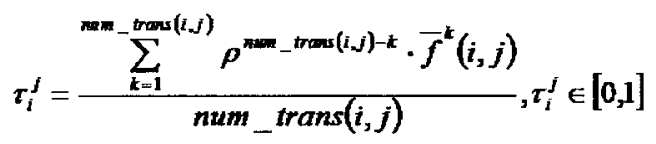

Where, num_trans $(i, j)$ denote the transaction time, $\rho(0<\rho<1)$ denote the time attenuation factor, and $\bar{f}^{k}(i, j)$ denote average reputation feedback. 
Definition $2 W S(j)=\left\{x \mid \exists x \in x_{k} \wedge x_{k} \in X\right\}$ denote the witness set of user $j$, user $x$ who satisfies $x \in x_{k} \wedge x \in W S(j)$ is defined as witness $x$ in trust path $x_{k}$.

Definition 3 If there exist a trust path $x=x\left(i, o_{1}, o_{2}, \cdots, o_{k}, \cdots, o_{n-1} j\right)$, of which $o_{k}$ is the $k$ th recommender in trust path $x$, then the direct trust $\tau_{i}^{j}\left(\tau_{j}^{i}\right)$, which user $i(j)$ holds about user $j(i)$ in trust path $x$, is given by

$$
\widetilde{\tau}_{j}^{j}=\prod_{k=1}^{I-1}\left[C R_{1}\left(o_{k}\right) \cdot C R_{2}\left(o_{k}\right) \cdot \tau_{a_{i-1}}^{a_{z}}\right] \cdot\left[C R_{2}\left(o_{i-1}\right) \cdot \tau_{a_{j-1}}^{y_{i}}\right] \widetilde{\tau}_{j}^{j} \in[0,1]
$$

$C R_{1}($.$) denote credibility of recommendation information, which is given by$

$$
\operatorname{Cre}(x)=\sqrt{\frac{(\lambda-1)^{2}+1-(l-1)^{2}}{(\lambda-1)^{2}+1}}, \lambda \geq l \geq 2
$$

Where, $\lambda$ denote the longest trust length, $l$ denote length of trust path $x$.

\subsection{Trust Path Deducing}

To evaluate trust degree of user $j$, user $i$ need search recommender of user $j$ within own trustable user set $T N(i)$ or selected recommender's trustable user set $T N($.$) first$ of all, then seek trust paths whose length is shorter than 6 between user $i$ and user $j$, finally computing the direct trust $\widetilde{\tau}_{i}^{J}$ that user $i$ holds about user $j$, according to formula (1). If length of trust path is bigger than 6 , there doesn't exist any trust path between user $i$ and user $j$, in other words there doesn't direct trust between user $i$ and user $j$. Algorithm 1 gives the implementation of above trust path deducing.

\subsection{Trust Path Combination}

Definition 4 Supposing that there exist $\mathrm{m}$ trust paths $x_{1}, x_{2}, \cdots, x_{m}$ between user $i$ and user $j$. If $x_{i} \cap x_{j} \neq \phi$, trust path $x_{i}$ and $x_{j}$ is defined as parallel trust path.

Currently the common means of trust path combination system mainly includes arithmetical average [1], weighted average [9] and weight based etc [10]. Abovementioned means all have limitations, such as excessively simple operation and overly sharp subjectivity while weight setting etc. In order to resolve abovementioned problem, the Beth model adopts equally deducing number to combine trust paths. However, it doesn't consider the distortion problem of recommended information while combing trust paths. Therefore it will bring about computing deviation of recommending trust. The paper introduces recommendation information credibility therefore and steers an improvement to the Beth model.

Let $x_{j}$ denote the jth trust path, the formula to combine trust path is given by

$$
V_{c o m}=\sqrt[\pi_{i}]{\prod_{j=1}^{n_{i}} \operatorname{Cre}\left(x_{j}\right) \cdot V_{i j}}
$$




\subsection{Unknown User Trust Computing}

Based on means proposed by Beth, the paper presents a modified means to compute unknown user's trust degree, by adopting the credibility of recommendation information. Direct trust $\tilde{\tau}_{c o m}(i, j)$ that user $i$ holds about unknown user $j$ is given by

$$
\tilde{\tau}_{c o m}(i, j)=1-\prod_{k \in \mathbb{P} S(j)} \sqrt[n_{k}]{\prod_{l=1}^{n_{k}} \operatorname{Cre}\left(x_{k, l}\right) \cdot\left(1-\tau_{i, k l}^{j}\right)}
$$

Where, $j . k . l$ denote witness $k$ of user $j, k . l$ denote the $l$ th trust path whose terminal node is $k, n_{k}$ denote the number of trust paths whose terminal node is $k$.

\section{Experiment}

We used Lucking-Reilly's eBay data set [11]. He collected 2439 transaction data between June $12^{\text {th }} 1999$ and August $4^{\text {th }}$ 1999. Table 1 shows the data description. There are 1026 unique users, which forms a virtual social networks $G$.

Table 1. Data Description

\begin{tabular}{llccc}
\hline Variable & Amount & $\begin{array}{c}\text { Average } \\
\text { amount of } \\
\text { transaction }\end{array}$ & $\begin{array}{c}\text { Average } \\
\text { transaction }\end{array}$ & $\begin{array}{c}\text { Average } \\
\text { transaction price } \\
(\$)\end{array}$ \\
\hline Buyer & 691 & 2.043 & 3.530 & 134.078 \\
Seller & 380 & 3.716 & 6.418 & 222.439 \\
Double as & 45 & & 4.8 & 249.832 \\
\hline
\end{tabular}

\subsection{Single User Experiment}

We choose a buyer named cobra76 from 691 buyers randomly. As shown in Fig. 1, comparing with Beth model, our model' error is smaller than Beth's. The reason is that our model computes trustworthiness. Our model can not only improve computing efficiency, but also overcome malicious recommenders' negative effect on trustworthiness computing. Moreover, when combining trust path, we incorporate credibility of trust path into our model to reduce computing error aroused by recommend information dissipation. Thus, it enhances our model's precision.

\subsection{Multi User Experiment}

We choose one buyer (or seller) for each credit rank from 691 buyers randomly, then use our model and Beth model to compute given users' trustworthiness separately and compare two models' error (as shown in Table 2 and Fig. 2). 


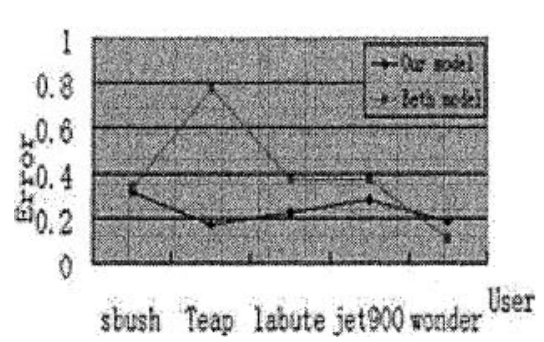

Fig. 1. Trustworthiness Computing Error

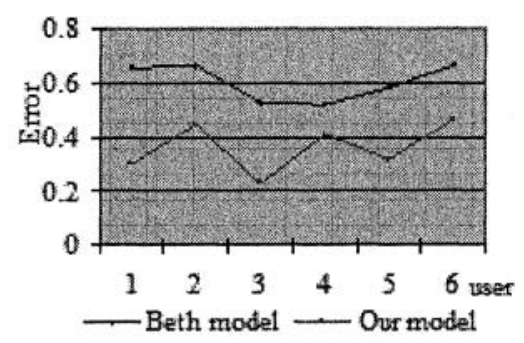

Fig. 2. Trustworthiness Computing Error

Table 2. Computing Error Comparison

\begin{tabular}{ccccccc}
\hline User & \multirow{2}{*}{ Chosen user } & Credit & \multicolumn{2}{c}{ Beth model } & \multicolumn{2}{c}{ Our model } \\
\cline { 5 - 6 } No. & & rank & Average error Total error Average error Total error \\
\hline 1 & shoalscoins & 1 & 0.6567 & 0.6011 & 0.3033 & 0.3624 \\
2 & juee & 2 & 0.6617 & & 0.4443 & \\
3 & goodealcoins & 3 & 0.525 & 0.2313 & \\
4 & epach29601@aol.com & 4 & 0.5222 & 0.4129 & \\
5 & bbfinethings & 5 & 0.5797 & 0.3182 & \\
6 & alscoin@home.com & 6 & 0.6612 & 0.4644 \\
\hline
\end{tabular}

As shown in table2, our model's total error is 36.24 , which is smaller than Beth's $(60.11 \%)$. It indicates that our model is more feasible and precise than Beth model. And the results demonstrate that building given users' social network is an effective method to measure trustworthiness about strangers.

\section{Conclusions}

Aiming at the scarcity of personality in existing trust model, utilizing the theory of social network and collaborative filtering, the paper designs a recommendationbased trust model. The later experiments indicate that comparing with Beth model, this model is available and feasible.

\section{Acknowledgements}

The National Natural Science Foundation of China under Grant No. 70371004 and the $\mathrm{PhD}$ Program Foundation of Education Ministry of China under Contract No. 20040006023 support this paper. 


\section{References}

1. E. Adarand and B. Huberman, Free Riding on Gnutella, Palo Alto: Xerox PARC Technical Report, CSL-00-3, 2000.

2. T. Beth, M. Borcherding, and B. Klein, Valuation of Trust in Open Network, Proceedings of the European Symposium on Research in Security, 3-18 (1994).

3. A. Abdul- Rahman and S. Hailes, Supporting Trust in Virtual Communities, Proceedings of the Hawaii International Conference on System Sciences 33, Jan 4-7 (2000).

4. F. Rino, P. Giovanni, and C. Cristiano, A Fuzzy Approach to a Belief-Based Trust Computation. Proceedings of the AAMAS 2002 International Workshop on Trust, Reputation, and Security: Theories and Practice, 73-86 (2002).

5. S M. Tanley, Six Degrees of Separation. http://weblab.unilueneburg.de/weblab/seminare/netze.

6. Z. Junmao, Y. Shoubao, and F. Jianping, A Grid\&P2P Trust Model Based on Recommendation Evidence Reasoning. http://www.chinagrid.net/dvnews/

7. B. $\mathrm{Yu}$ and $\mathrm{M}$. Singh, Trust and reputation management in a small-world network, Proceedings of the 4th International Conference on MultiAgent Systems, 449-450 (2000).

8. A. Jøsang and S. Hird, Simulating the Effect of Reputation Systems on e-Markets, Proceedings of the First International Conference on Trust Management, 179-194 (2003).

9. G. Zacharia, Collaborative Reputation Mechanisms for Electronic Marketplaces, Decision Support Systems 29, 371-388 (2000).

10.R. Guha, R. Kumar, and P. Raghavan, Propagation of trust and distrust, Proceedings of the 2004 International Conference on the WWW, 403-412 (2004).

11. D. Lucking-Reiley, Pennies from eBay: The Determinants of Price in Online Auctions, http://www.vanderbilt.edu/econ/reiley/papers/PenniesFromEBay.pdf. 